Check for updates

Cite this: RSC Adv., 2017, 7, 18340
Received 27th December 2016 Accepted 14th March 2017

DOI: $10.1039 / c 6 r a 28742 e$

rsc.li/rsc-advances

\section{Photoluminescence from amino functionalized graphene quantum dots prepared by electrochemical exfoliation method in the presence of ammonium ions $\uparrow$}

\begin{abstract}
Chien-Te Hsieh, (D) * Dong-Ying Tzou, Keng-Yen Hsieh and Ken-Ming Yin
This study elucidates how the nitrogen functionalities affect the UV-visible absorption and photoluminescence (PL) emission of N-modified graphene quantum dots (NGQDs). An electrochemical exfoliation technique incorporated with an in situ amino-functionalization is adopted to synthesize $\mathrm{N}$ modified graphene nanosheets (GNs) with different amidation levels. The applied voltage plays a crucial role in tuning the N/C atomic ratio on the GN product. The maximal N/C atomic ratio reaches $8.4 \%$ when exfoliating the GNs at $5 \mathrm{~V}$. The nitrogen functionalities on the GNs include (i) amide/amino functional groups, decorated to the edge sites of GNs, and (ii) pyridinic/pyrrolic/quaternary N, planar to the GNs. The tunable PL emission is strongly affected by the $n-\pi^{*}$ transitions, forming an interstate transition. The $\mathrm{N}$ chemical doping in $\mathrm{C}=\mathrm{C} \mathrm{sp}^{2}$ domains and amino edge functional groups strongly influences the bonding and anti-bonding of molecular orbits, leading to different electronic transition $\mathrm{n}$ states. Thus, the $\mathrm{N}$ dopant and edge $\mathrm{N}$ functionalities serve as new radiative sites to tune the $\mathrm{PL}$ emission. The surface coverage of $\mathrm{sp}^{2}$ carbon bonding decreased with an increase in the number of $\mathrm{N}$ functional groups, inducing a red shift. Therefore, the color-tunable emission from blue to greenish color and the increase of absorption in the visible region can be done by tuning appropriate amidation level on the NGQD specimens.
\end{abstract}

\section{Introduction}

Graphene is a monolayer of carbon atoms tightly arranged into a two-dimensional honeycomb lattice, ${ }^{1}$ offering a wide range of applications including transparent conducting films, ${ }^{2}$ catalyst supports, ${ }^{3}$ lithium-ion batteries, ${ }^{4}$ and electrochemical capacitors ${ }^{5}$ because of its extraordinary mechanical, electronic and optical properties. The other derivative, graphene oxide (GO), is a chemically modified graphene, decorated with oxygen functionalities such as hydroxyl, carbonyl, and carboxyl groups. It is generally recognized that pristine graphene exhibits a high electron mobility of $10^{-6} \mathrm{~cm}^{2} \mathrm{~V}^{-1} \mathrm{~s}^{-1}$ and an almost zero band gap, ${ }^{6}$ whereas the band gap and optical properties of GO sheets strongly depend on their oxidation levels. ${ }^{7}$ This finding raises great interest in surface modification of graphene which enables tuning of the optical and electronic properties for practical applications. Recently, the chemical doping of GNs with heteroatoms like sulfur, boron and nitrogen has emerged due to its ability to provide

Department of Chemical Engineering and Materials Science, Yuan Ze University, Taoyuan 32003, Taiwan. E-mail: cthsieh@saturn.yzu.edu.tw; Fax: +886-3-4559373; Tel: $+886-3-4638800$ ext. 2577

$\dagger$ Electronic supplementary information (ESI) available. See DOI: 10.1039/c6ra28742e fascinating optical and electronic characteristics. ${ }^{8-10}$ Both dopant type and concentration have been considered as crucial factors to alter the band structure of graphene, i.e., the energy gap between valence and conduction bands. ${ }^{10}$ Particularly, nitrogen-doping in carbon delivers a very promising potential in a variety of applications such as graphene quantum dots (GQDs). ${ }^{11,12}$ The presence of $\mathrm{N}$ atoms has been confirmed to tailor the charge density on carbon, originated from an incorporation of $\mathrm{N}$ atoms with excess electrons, i.e., n-type semiconductor. ${ }^{13}$ Recently, a number of methods have been developed to prepare N-doped GNs or GO sheets, including $\mathrm{NH}_{3}$ annealing followed by $\mathrm{N}^{+}$-ion irradiation, ${ }^{10}$ hydrothermal reaction with ammonia, ${ }^{9,11}$ ammonia-ultrasonic bathing method, ${ }^{12}$ chemical doping of epitaxial graphene by 4 amino-2,2,6,6-tetramethyl-1-piperidinyloxy, ${ }^{14}$ and chemical doping of trans- and cis-azobenzene.$^{15}$ Usually, the $\mathrm{N}$ functionalization (i.e., N-doping or chemical decoration) on GNs are carried out after the preparation of GO sheets. There are few reports focusing on one-step processes, consisted of graphene synthesis and amino-functionalization.

This work intends to adopt one-step process, incorporated with $\mathrm{N}$-functionalization and electrochemical exfoliation $(\mathrm{N}$ $\mathrm{EE})$, to synthesize high quality GN products. Basically, the EE method offers great potential in large-scale production due to 
its high efficiency and better environmental friendliness, as compared to other ones (e.g., Hummers' method and chemical vapor deposition). ${ }^{\mathbf{1 6 - 1 9}}$ Moreover, as the exfoliation of graphite takes place in aqueous electrolyte at ambient temperature, the overall process is economic. In comparison, the N-EE approach could be operated in $\left(\mathrm{NH}_{4}\right)_{2} \mathrm{HPO}_{4}$, forming $\mathrm{N}$-modified GN products without any further modification. The voltage, ranged from 5 to $12 \mathrm{~V}$, serves as an operating parameter to tune the amidation level and amino group distribution on GN powders. Meanwhile, the exfoliation and $\mathrm{N}$-doping could take place simultaneously, shortening operation period and thus showing great feasibility for commercialization. To explore their photoluminescence (PL) performance, the amino-functionalized GNs could be sieved and classified within the region of 3-5 $\mathrm{nm}$ in particle size through a high-performance centrifugal process, thereby giving N-modified GQDs (NGQDs). In fact, pioneering studies have reported one possibility of tailoring the PL emission of GQDs, strongly influenced by their size, morphology and surface functionalization. ${ }^{20-23}$ However, an understanding regarding the PL emission from NGQDs prepared by one-step NEE route is still unclear. Accordingly, this work aims to clarify the PL emission from the NGQDs, prepared through the N-EE route. The relationship between the band gap and the PL efficiency of NGQDs is systematically investigated. The present work explores one efficient protocol that employs one-step N-EE method followed by high-performance nano-sieving process to synthesize the NGQDs with different amidation levels, using $\left(\mathrm{NH}_{4}\right)_{2} \mathrm{HPO}_{4}$ electrolytes under the constant-voltage within potential range of $5-12 \mathrm{~V}$.

\section{Experimental}

\section{Fabrication of NGQDs by N-EE method}

The N-EE method for producing $\mathrm{N}$-functionalized GNs in a large quantity could be briefly described as follows. First of all, two pieces of highly-oriented pyrolytic graphite (HOPG) plates were carefully cut into a dimension of $10 \times 10 \times 2 \mathrm{~cm}^{3}$. We adopted one two-electrode configuration system, consisted of 15 liter Teflon tank and in-serial five channels, to fabricate GN products in pilot scale. Thus, the N-EE approach enabled mass-production of GNs from ten pieces of HOPG electrodes simultaneously when operating at high voltage. The distance between each HOPG electrode was set at $3 \mathrm{~cm}$, and the electrolyte were prepared using $10 \mathrm{~mol} \%\left(\mathrm{NH}_{4}\right)_{2} \mathrm{HPO}_{4}$. The N-EE process was carried out by applying two-step DC bias on the HOPG electrodes. In the first-step a potential difference of $1 \mathrm{~V}$ was maintained for $0.2 \mathrm{~h}$; and in the second step the potential difference is raised to different values (i.e., 5, 7, 9, and $12 \mathrm{~V}$ ) and then maintained at the same voltages for $0.5 \mathrm{~h}$. After the N-EE process, the GN flakes were collected and dried at $105{ }^{\circ} \mathrm{C}$ in one vacuum oven overnight. The collection of $\mathrm{N}$-functionalized GQDs was described as follows. A highperformance centrifuge was used to sieve and screen the GN powders into various particle sizes, using polymeric filters with different nominal pore sizes. The rotation speed and centrifugation period were set at $10000 \mathrm{rpm}$ and $0.5 \mathrm{~h}$, respectively.

\section{Characterization of N-modified GNs and NGQDS}

HR-TEM (JEOL, JEM-2100) was adopted to inspect the microstructural GN powders. FTIR (Nicolet Avatar 360 FTIR) spectroscopy was adopted to identify surface functional groups on graphene samples. Herein 32 scans in the spectral ranges were recorded with a resolution of $4 \mathrm{~cm}^{-1}$. We employed X-ray photoelectron spectroscopy (XPS) to characterize the chemical composition of N-modified GN powders. Each XP spectrum was recorded by a Fison VG ESCA210 spectrometer with $\mathrm{Mg}-\mathrm{K} \alpha$ radiation. Both the $\mathrm{C}$ 1s and $\mathrm{N}$ 1s spectra were deconvoluted by using a non-linear least squares fitting program with a symmetric Gaussian function. The surface composition of asprepared GN samples was calculated with an appropriate sensitivity factor.

The particle size distributions of as-prepared NGQD samples were analyzed by AFM (Ardic P100). The ultraviolet-visible (UVvis) absorbance spectra of GN suspension (weight ratio: $0.05 \mathrm{mg}$ GN/1 mL distilled water) in quartz cell were analyzed and collected using an UV-vis spectrometer (Agilent Technology Cary 60) with a wavelength scan rate of $60 \mathrm{~nm} \mathrm{~min}^{-1}$. Herein the distilled water served as a blank spectrum. For the PL characterization, the emission and excitation spectra of GN suspensions were recorded by using an optic spectrometer (FLS920P Edinburgh Analytical Instrument), equipped with Xe lamp as an excitation source. We set the wavelength of excitation at $360 \mathrm{~nm}$. Herein all GN powders were dispersed in distilled water to avoid re-absorption and other interferences. The PL measurements were performed at room temperature.

\section{Results and discussion}

The as-prepared GN samples were designated to GN-5, GN-7, GN-9, and GN-12, according to the applied voltages: 5, 7, 9, and $12 \mathrm{~V}$, respectively. After the size classification, the GN samples were collected and designated as NGQD-5, NGQD-7, NGQD-9, and NGDQ-12, also based on the applied voltages: 5, 7, 9, and $12 \mathrm{~V}$, respectively. Fig. 1(a)-(d) present high-resolution
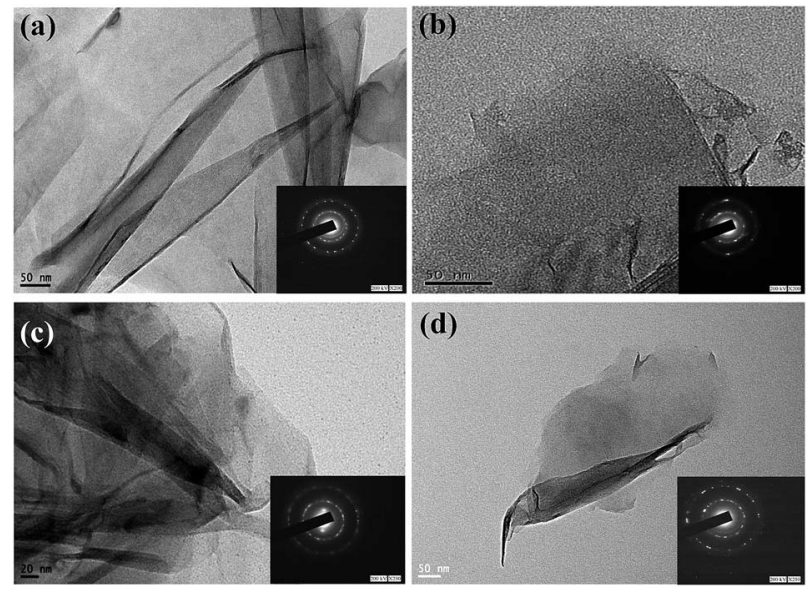

Fig. 1 HR-TEM micrographs of (a) GN-P5, (b) GN-P7, (c) GN-P9, and (d) GN-P12. The inset shows the SAD pattern for each amino-functionalized GN products, prepared by N-EE method. 
transmission electron microscope (HR-TEM) micrographs of Nmodified GNs, and the insets depict the selected-area diffraction (SAD) patterns for all samples. The atomic force microscope (AFM) images and their size-distribution histograms are presented in Fig. S1 in the ESI. $\uparrow$ The layer number, observed from AFM micrographs, ranges from 6 to 10. The HR-TEM images reveal that the as-prepared GNs have a curved morphology with several micrometers in length. Each GN sample is found to have a few stacked graphene layers. There are several rings in the SAD patterns, confirming the existence of polycrystalline structures in each GN sample. The production yield of $\mathrm{N}$-functionalized GN powders could be evaluated by the ratio of gain of GNs to loss of graphite electrode. The average yield for all operating voltages is approximately $80 \%$. As observed from AFM images, all NGQD samples display similar particle size distributions, in the range of 3-5 $\mathrm{nm}$. Fig. 2(a) presents the Fourier transformed infrared (FTIR) spectra of different NGQDs, showing the presence of oxygen and amino functional groups on the NGQD samples. The spectra of NGQDs exhibit characteristic bands around 1410 and $1730 \mathrm{~cm}^{-1}$ for tertiary alcoholic $\mathrm{C}-\mathrm{OH}$ bending and $\mathrm{HO}-\mathrm{C}=\mathrm{O} / \mathrm{C}=\mathrm{O}$ stretching, respectively. The transmittance bands at $1100 \mathrm{~cm}^{-1}$ can be assigned to $\mathrm{C}-\mathrm{N}$ stretching vibrations. ${ }^{24}$ It is worth noting that the $\mathrm{O}-\mathrm{H}$ group at $3300-3500 \mathrm{~cm}^{-1}$ corresponds to physically
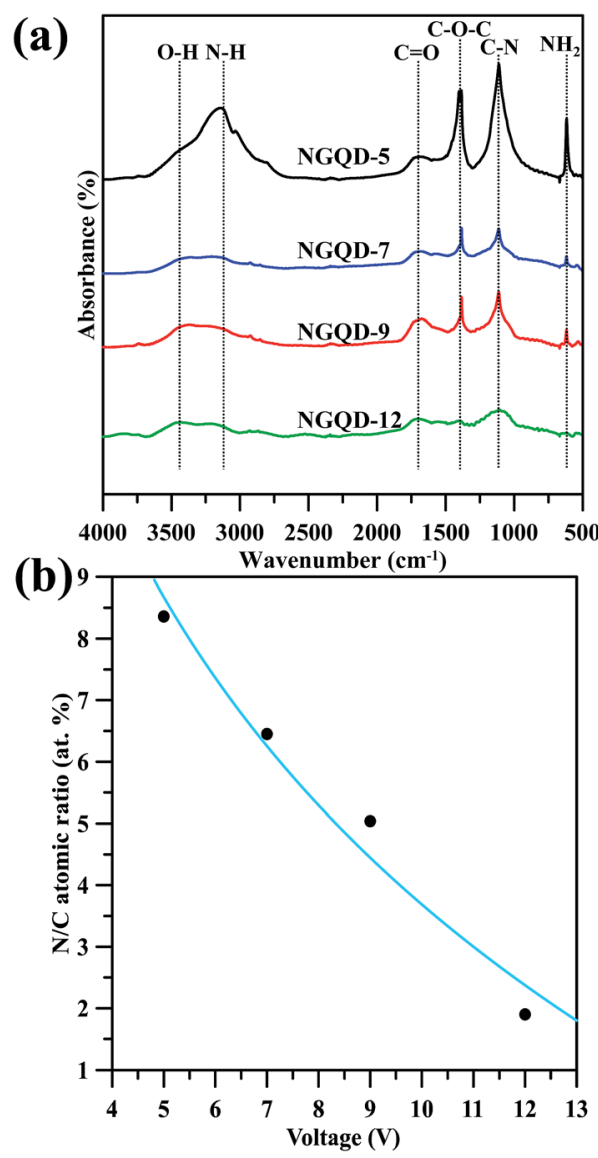

Fig. 2 (a) FT-IR transmittance spectra of amino-functionalized GN products prepared at different voltages. (b) The N/C atomic ratio as a function of applied voltage. adsorbed moisture onto carbon surface, while the band appears at $2900-3200 \mathrm{~cm}^{-1}$ for the vibration of $\mathrm{N}-\mathrm{H}$ groups in $\mathrm{sp}^{2}$ domain plane of GNs. ${ }^{25}$ The NGQD-5 sample shows an emerging band in the range of $2900-3200 \mathrm{~cm}^{-1}$ as compared to the others, indicating presence of a large amount of amino or amide groups. Accordingly, the N-EE technique operated at low voltage is able to produce GN product with low amidation level. This result is also in accordance with the pioneering study, ${ }^{26}$ in which the $\mathrm{N}$ chemical doping is accompanied by the introduction of oxygen-containing functionalities, e.g., hydroxyl, carbonyl and carboxyl groups.

The full-range XPS spectra of N-modified GN sample are illustrated in Fig. S2 (see ESI $\dagger$ ), showing the $\mathrm{C} 1 \mathrm{~s}, \mathrm{~N} 1 \mathrm{~s}$, and $\mathrm{O} 1 \mathrm{~s}$ peaks at binding energies of approximately 284,400 , and $532 \mathrm{eV}$, respectively. The quantitative analysis of the XPS spectra reflects that the GN-P5 sample exhibits the high N/C atomic ratio of $8.4 \%$ among the GN samples. The N/C atomic ratio as a decreasing function of applied voltage is depicted in Fig. 2(b). This result reveals that the exfoliation of GNs in $\left(\mathrm{NH}_{4}\right)_{2} \mathrm{HPO}_{4}$ at $5 \mathrm{~V}$ sequentially implant nitrogen atoms and attach amino groups to the GNs, while the exfoliation at $12 \mathrm{~V}$ is prone to dramatically separate the GNs from the graphite electrodes with little $\mathrm{N}$ chemical doping and less implantation of $\mathrm{N}$-containing functional groups. Therefore, it can be said that, this approach provides an efficient pathway to produce N-modified GN product through an electrochemical exfoliation incorporated with in situ amino-functionalization.

The proposed mechanism concerning N-EE approach illustrated in Fig. 3 consists of the following steps: (i) ionic intercalation, (ii) graphite layer expansion, and (iii) bubble expansion. In the step (i), water is initially oxidized to generate hydroxyl $\left(\mathrm{OH}^{*}\right)$ and oxygen $\left(\mathrm{O}^{*}\right)$ radicals at high potentials (i.e., 5-12 $\mathrm{V}$ in this case), leading to the corrosion of edges sites and grain boundaries and opening up some defective sites of expanded graphite. ${ }^{19,27,28}$ In the step (ii), the oxygenated graphite layers tend to be expanded, allowing ionic intercalation followed by surface oxidation/amidation in the presence of various ions, e.g., $\mathrm{OH}^{-}$, $\mathrm{NH}^{4+}, \mathrm{H}^{+}$, and $\mathrm{PO}_{4}{ }^{3-}$. The step (iii) enables the anion reduction

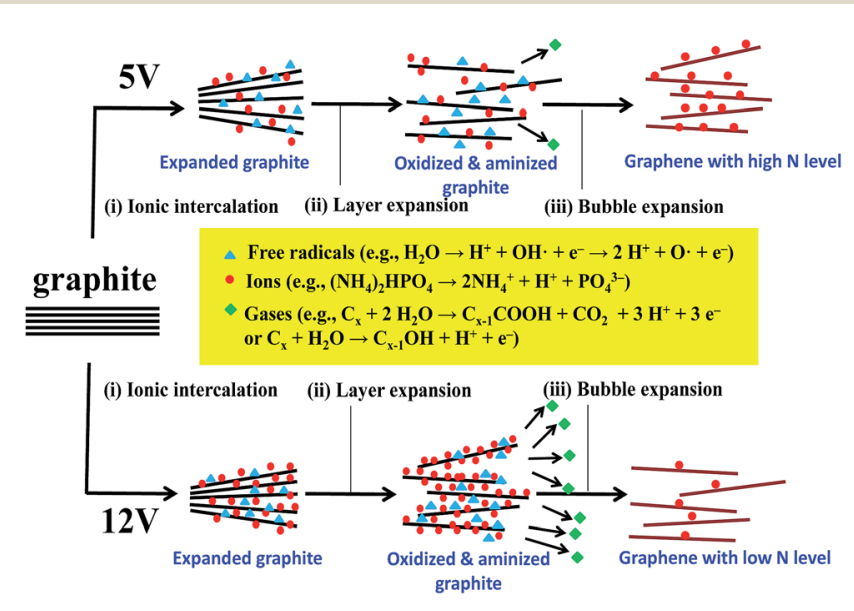

Fig. 3 Schematic illustration for the electrochemical exfoliation incorporated with amino-functionalization method of producing graphene nanosheets with different amidation levels. 
and water oxidation, inducing the release of $\mathrm{O}_{2}$ and other gases $\left(e . g\right.$., $\left.\mathrm{CO}_{2}\right) \cdot{ }^{27,28}$ The bubble expansion thus makes the exfoliation of graphene layers from the HOPG electrodes. Meanwhile, there are a large number of dangling bonds generated on graphene sheets in the presence of $\mathrm{NH}^{4+}$, in which nitrogen dopants would chemically interact with the defect sites and amino groups tend to terminate the dangling bond on the edges sites of graphene layer. Thus, the N-EE method leads to an in situ aminofunctionalization route during the electrochemical exfoliation process. When operating at $12 \mathrm{~V}$, the chemical kinetics of water electrolysis becomes faster, thus leading high exfoliation rate, i.e., high production rate of GNs. Herein, the in situ aminofunctionalization cannot take place completely due to its higher energy barrier of forming amino group and $\mathrm{N}$ chemical doping, compared to the exfoliation rate of GNs. At present, the rate of generation of oxygen functionalities, mainly originated from the free radicals (i.e., hydroxyl and oxygen radicals), is much higher than that of amino and amine groups on N-modified GNs. Thus, the N-modified GNs show higher amidation level when exfoliating at low voltage (e.g., $5 \mathrm{~V}$ ). The related chemical reaction steps regarding the formation mechanism have been formulated and expressed in Fig. 3.

Fig. 4 presents the focused C 1s spectra of N-modified GN samples, composed of several peaks obtained by deconvolution: C-C (284.6 eV), C-N (285.8 eV), C-OH $(286.5 \mathrm{eV}), \mathrm{C}=\mathrm{O}$ $(288.0 \mathrm{eV})$, and $\mathrm{O}-\mathrm{C}=\mathrm{O}(289.1 \mathrm{eV}) .{ }^{9,24}$ The later three oxygen functionalities, phenolic, carbonyl, and carboxylic group, are frequently attached to basal and edge sites of graphene layer. It should be noted that the emergence of $\pi-\pi^{*}$ satellite peak $(\sim 298 \mathrm{eV})$ of $\mathrm{sp}^{2}$ carbon domain in all N-modified GN samples confirms the resonance in the aromatic orbitals of GNs. It can
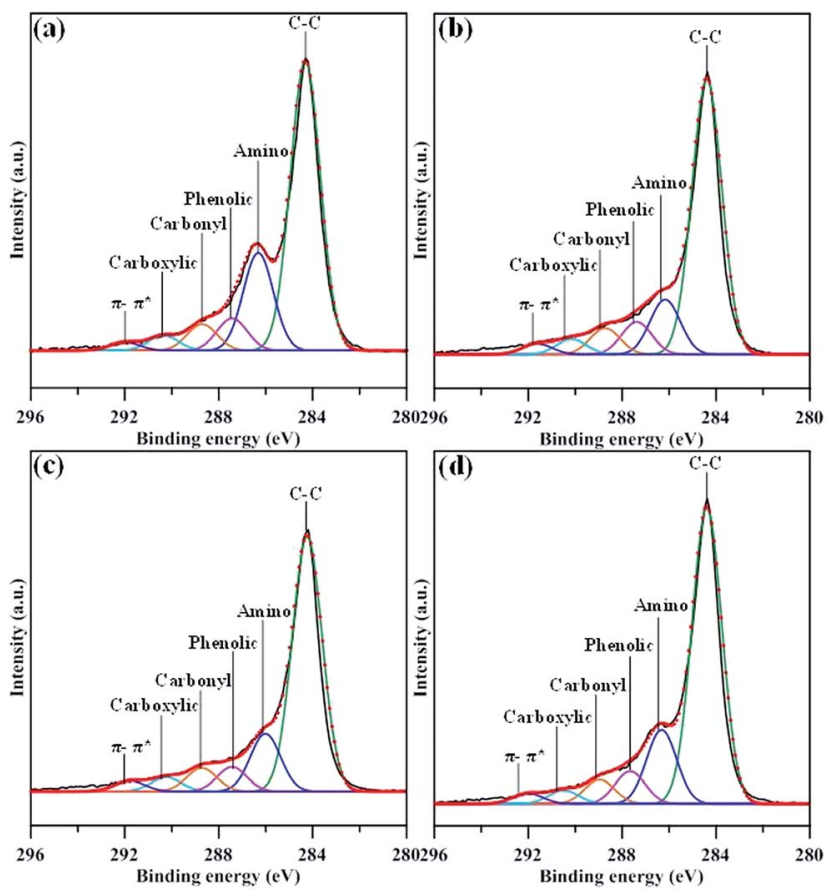

Fig. 4 XPS $C$ 1s peaks of amino-functionalized GN products: (a) GNP5, (b) GN-P7, (c) GN-P9, and (d) GN-P12. be seen that the GN-P5 sample offers the largest C-N intensity, showing the highest amidation level as compared to the other three samples. This reflects that the in situ aminofunctionalization increases the contribution of $\mathrm{C}-\mathrm{N}$ bond at the expense of that of oxygen functionality, e.g., carbonyl and carboxylic groups. The conversion of the nitrogen functionality caused by the in situ amino functionalization route can also be elucidated by the analysis of $\mathrm{N} 1 \mathrm{~s}$ spectra.

Fig. 5 depicts the $\mathrm{N}$ 1s spectra of $\mathrm{N}$-modified GN samples, comprising five main peaks assigned to pyridinic $(\sim 398.4 \mathrm{eV})$, amino $\left(-\mathrm{NH}_{2}, 399.2 \mathrm{eV}\right)$, pyrrolic $(\sim 399.6 \mathrm{eV})$, quaternary $(\sim 400.7$ $\mathrm{eV})$, and amide $(\mathrm{O}=\mathrm{C}-\mathrm{N}, 401.2 \mathrm{eV})$ functional groups. ${ }^{29,30}$ The composition of nitrogen functionalities for all N-modified GN samples, determined from the deconvolution of $\mathrm{N}$ 1s peak using a multiple Gaussian function. The presence of pyridinic, pyrrolic, quaternary functionalities on GNs reveals that $\mathrm{N}$ atoms are doped through replacing $\mathrm{C}$ atoms of graphene $\mathrm{e}^{24}$ through the N-EE route. The quaternary $\mathrm{N}$ atoms doped in aromatic graphene lattice could repair the vacancy defects in graphene sheets. ${ }^{31,32}$ It can be observed from Fig. 5 that the percentage of amide group delivers an increasing trend after exfoliating at $7-12 \mathrm{~V}$. We believe that the amide functionalities were generated in large amounts at the expense of loss of pyridinic and pyrrolic nitrogen or the replacement of carboxylic groups. The formation of amide may be associated with the cleavage of $\mathrm{C}-\mathrm{N}$ bonds in pyridinic and pyrrolic groups ${ }^{24}$ or the strong interaction between ammonia and oxygen functionalities during the exfoliation incorporated with in situ $\mathrm{N}$-functionalization process.

Fig. 6 shows ultraviolet (UV)-visible spectra of NGQD samples in distilled water, in which the maximal absorbance can be attributed to $\pi-\pi^{*}$ transition in $\mathrm{sp}^{2}$ domains, whereas
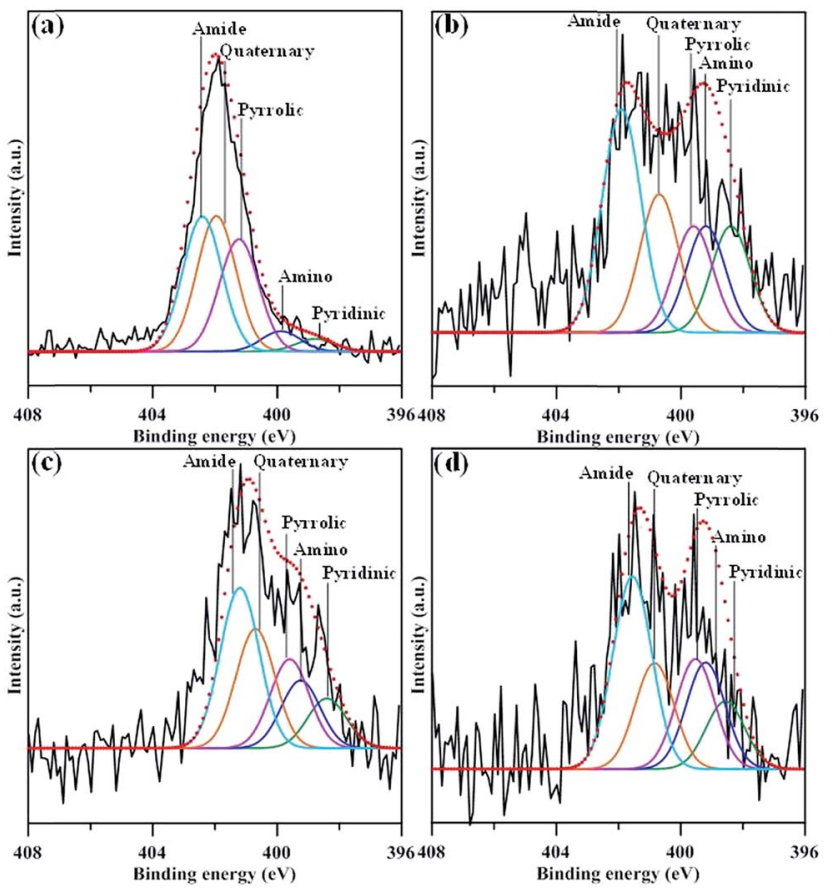

Fig. 5 XPS N 1s peaks of amino-functionalized GN products: (a) GNP5, (b) GN-P7, (c) GN-P9, and (d) GN-P12. 
the absorption edge mainly originates from $n-\pi *$ transitions of nonbonding electrons of adatoms, ${ }^{33-35}$ e.g., nitrogen functionalities. Apparently, there is an obvious shift of adsorption band edge to long wavelengths with an increase in the N/C atomic ratio of NGQDs. This shift is ascribed to the surface defect sites on NGQDs (i.e., nitrogen functionalities), thus, providing intermediate states (i.e., n-states) within the $\pi-\pi^{*}$ energy gap. Accordingly, the presence of nitrogen functionalities causes the red shift in UV light absorption, i.e., $\mathrm{n}-\pi^{*}$ transitions. The PL excitation spectra of NGQDs a also illustrated in Fig. 6. The feature of PL excitation spectra can be divided into three adsorptive transition regions: (i) $n-\pi^{*}$, (ii) $\pi-\pi^{*}$ and (iii) $n-\sigma$ transitions. $^{33}$ The maximal peak of the PL excitation for all NGQDs falls into the range of 405-415 $\mathrm{nm}$. The NGQD-5 displays the highest intensity of PL excitation as compared to the other three samples, indicating the importance of N/C atomic ratio and nitrogen group distribution. Because of all NGQDs with a narrow particle size distribution (see Fig. S1†), the PL excitation shift can be inferred from the number and location of carbon vacancy defects. When forming the carbon vacancies at the edges of NGQD with lower formation energy, the NGQDs would permit an efficient elastic relaxation of atoms for stress release (i.e., weak confinement). If these defects exist in the interior of NGQDs, the excitons require an extra energy to escape from the inner perfect crystal structure, leading to a larger lattice relaxation (i.e., strong confinement). ${ }^{6}$ Since the NQGD-5 sample possesses a large amount of nitrogen edge sites (i.e., amino/amide groups), the PL excitation tends to be a weak confinement behavior due to the transition of bound excitons localized inside the edge of N-modified GQDs. Thus, the weak confinement of excitons in the NGQDs imparts the high-energy PL emission. In contrast, the nitrogen functionalities, e.g.,
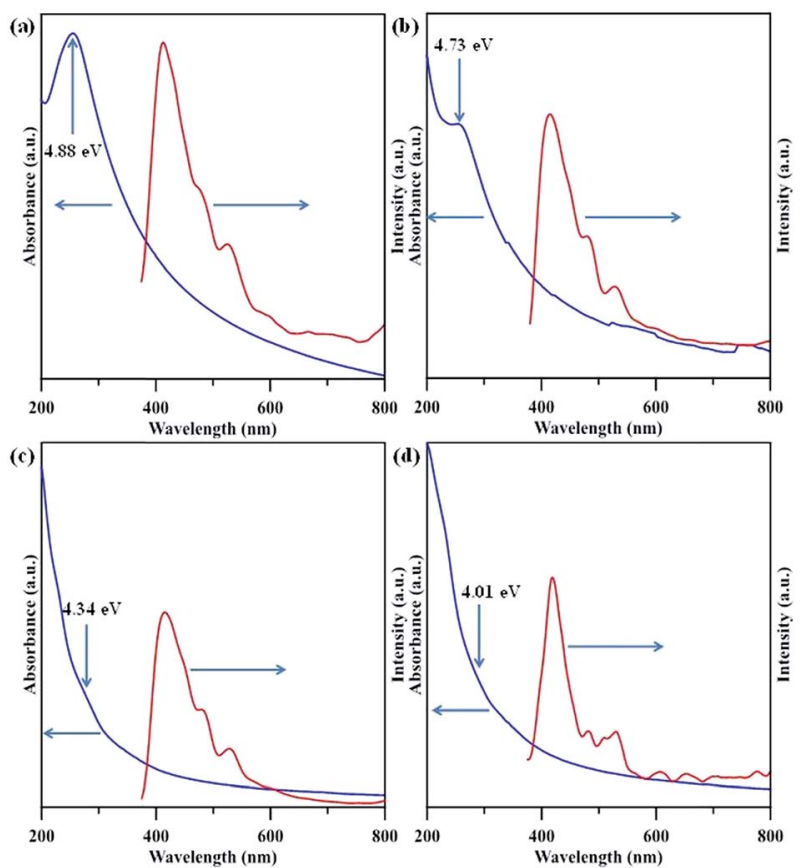

Fig. 6 UV-visible and PLE spectra of amino-functionalized GQDs: (a) NGQD-5, (b) NGQD-7, (c) NGQD-9, and (d) NGDQ-12. pyridinic- $\mathrm{N}$, pyrrolic- $\mathrm{N}$, quaternary- $\mathrm{N}$, is prone to tightly confine the excitons inside the interior of NQGDs, leading to a lower PL response.

Fig. 7(a) shows PL spectra of NGQD specimens excited at a wavelength of $340 \mathrm{~nm}$. The NGQDs display single PL response at $562 \mathrm{~nm}$ (NGQD-5), $557 \mathrm{~nm}$ (NGQD-7), $553 \mathrm{~nm}$ (NGQD-9), and $551 \mathrm{~nm}$ (NGQD-12). The red shift in the PL spectra with an increase in N/C atomic ratio can be viewed, encompassing the blue to green-blue of visible spectrum, as shown in Fig. 7(b). It is worthwhile to note that the NGQD-5 specimen is capable of offering the highest intensity of PL emission among the specimens. This is presumably due to one possible reason that the design of NGQD specimen displays the weak quantum confinement with increasing amino-functionalization extent. Concerning the shape of PL response, two types of bound excitons may exist in the NGQDs: (i) one is the bound excitons into NGQDs, contributing the low-energy transition, and (ii) the other is the bound excitons in edge sites of NGQDs, exhibiting the high-energy transition. This argument can be supported by the full width at half maximum (FWHM) of each PL peak. The FWHM for all PL responses shows an order as $118 \mathrm{~nm}$ (NGQD-5) $>112 \mathrm{~nm}$ (NGQD-7) > $96 \mathrm{~nm}$ (NGQD-9) > $91 \mathrm{~nm}$ (NGQD-12). Since the NQGD-5 specimen owns two different bound excitons, the NGQD-5 sample displays the wide PL emission from visible to near-infrared wavelength. Accordingly, this is an essence why the NGQDs with high amidation level offer highintensity fluorescence and broadened PL response.

Fig. 8(a) gives a schematic of energy levels associated with absorption and PL emission of NGQDs. Obviously, the tunable PL emission is strongly affected by the $n-\pi *$ transitions, forming an interstate transition. This is because the $\mathrm{N}$ chemical doping in $\mathrm{C}=\mathrm{C} \mathrm{sp}^{2}$ domains and amino edge functional groups influence the bonding and anti-bonding of molecular orbits, imparting different electronic transition n-states. ${ }^{12}$ The electron transition from $\pi^{*}$ orbital to $n$-state orbital consists of radiative recombination and phonon scattering. It is believed that the $\mathrm{N}$ dopant and edge $\mathrm{N}$ functionalities could act as new radiative sites to tune the PL emission. ${ }^{36,37}$ As for the NGQDs with low amidation level, the blue light emission to the intrinsic states associated with isolated $\mathrm{sp}^{2}$ nanodomains appears, whereas the greenish light illuminates from the NGQDs with high

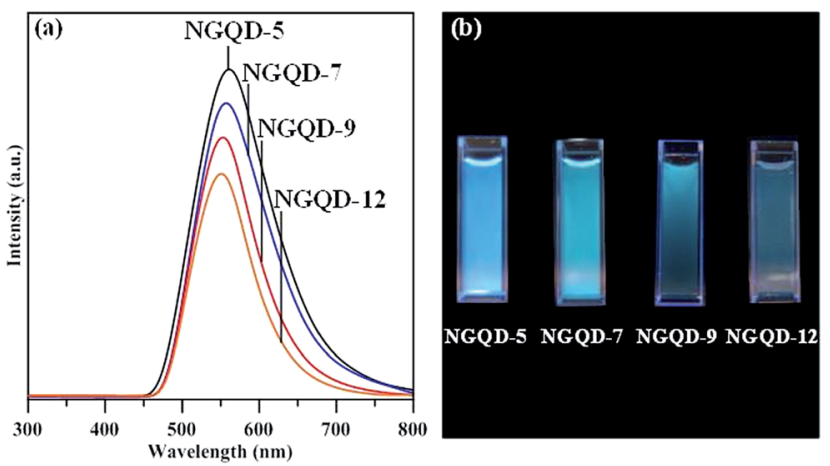

Fig. 7 (a) PL spectra and (b) photographs of amino-functionalized GQDs. 

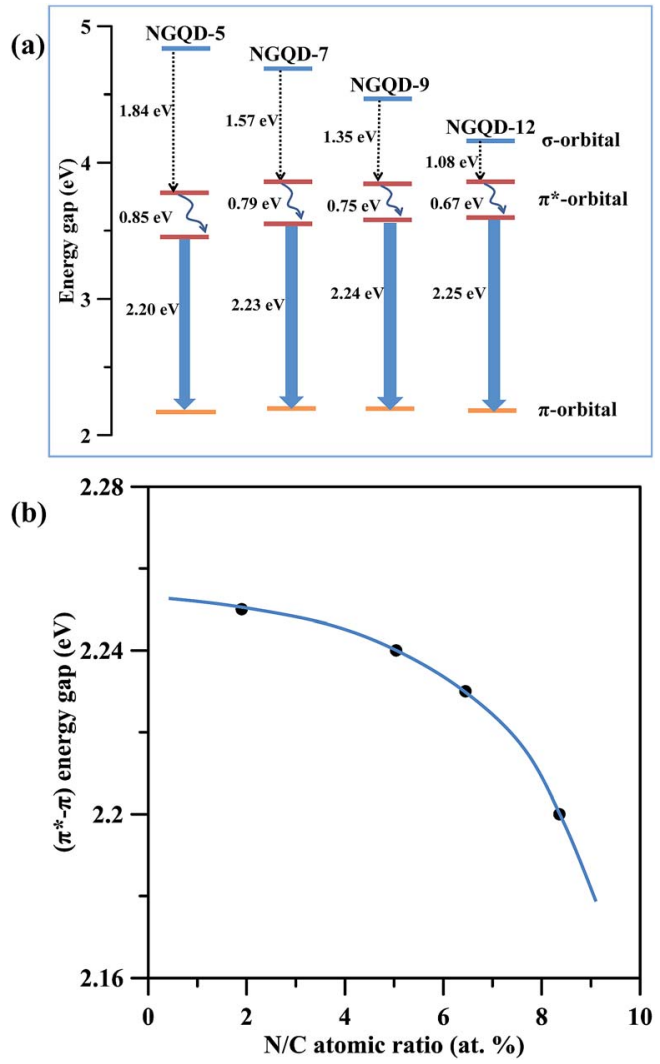

Fig. 8 (a) Band gap structure of different N-modified GQDs. (b) The $\left(\pi^{*}-\pi\right)$ energy gap in PL spectra as a function of N/C atomic ratio.

amidation extent due to the extrinsic states formed by different $\mathrm{N}$ functionalities. With increasing the amount of nitrogen functional groups, the surface coverage of $\mathrm{sp}^{2}$ carbon bonding decreases, thus leading to the red shift.

Therefore, the higher the amidation level, the suppressed the $\pi^{*}-\pi$ transition becomes, as illustrated in Fig. 8(b). In contrast, the $\pi^{*}-\pi$ transition becomes broader at low amidation level on NGQDs, showing a blue shift. The above achievement of the present work delivers a great potential that both the tunable PL emission and the increase of absorption in visible region are workable if tuning to an appropriate amidation level on the GQD specimens. Accordingly, this study provides a straightforward route to versatile applications in biosensors, optoelectronics, photodetection, photovoltaics, light-emitting diodes and plasmonics.

\section{Conclusions}

This study elucidated how the nitrogen functionalities affect the UV-visible absorption and PL emission of N-modified GQDs. An efficient exfoliation technique incorporated with an in situ aminofunctionalization was adopted to synthesize N-modified GNs with different amidation levels. The applied voltage served as a crucial factor in tuning the N/C atomic ratio on the GN product. The N/C atomic ratio of as-prepared GNs was a decreasing function of applied voltage, and the maximal $\mathrm{N} / \mathrm{C}$ atomic ratio reached to $8.4 \%$ when exfoliating the GN sample at $5 \mathrm{~V}$.
Analyzed by XPS, the nitrogen functionalities on the GNs include (i) amide/amino functional groups, decorated at the edge sites of GNs, and (ii) pyridinic/pyrrolic/quaternary $\mathrm{N}$, planar to the GNs. An obvious shift of UV adsorption band edge to long wavelengths was observed when increasing the $\mathrm{N} / \mathrm{C}$ atomic ratio of NGQDs. This shift was attributed to the surface defect sites on NGQDs (i.e., nitrogen functionalities), thus, providing intermediate states (i.e., n-states) within the $\pi-$ $\pi^{*}$ energy gap. Accordingly, the presence of nitrogen functionalities resulted in the red shift in UV light absorption, i.e., $\mathrm{n}-\pi^{*}$ transitions. Due to a large amount of nitrogen edge sites (i.e., amino/amide groups), the PL excitation tends to be a weak confinement behavior due to the transition of bound excitons localized inside the edge of N-modified GQDs. Thus, the weak confinement of excitons in the NGQDs facilitated the highenergy PL emission. In comparison, the nitrogen functionalities including pyridinic- $\mathrm{N}$, pyrrolic- $\mathrm{N}$, quaternary- $\mathrm{N}$, tended to tightly confine the excitons in the interior of NQGDs, imparting a lower PL response. The wide PL emission from visible to nearinfrared wavelength could be ascribed to two different bound excitons. A schematic of energy levels associated with absorption and PL emission of NGQDs was proposed. The tunable PL emission is strongly affected by the $n-\pi^{*}$ transitions, forming an interstate transition. This is because the $\mathrm{N}$ chemical doping in $\mathrm{C}=\mathrm{C} \mathrm{sp}^{2}$ domains and amino edge functional groups influence the bonding and anti-bonding of molecular orbits, imparting different electronic transition n-states. In our opinion the $\mathrm{N}$ dopant and edge $\mathrm{N}$ functionalities act as new radiative sites to tune the PL emission. As for the NGQDs with low amidation level, the blue light emission to the intrinsic states associated with isolated $\mathrm{sp}^{2}$ nanodomains appeared, whereas the greenish light illuminated from the NGQDs with high amidation extent due to the extrinsic states formed by different $\mathrm{N}$ functionalities. The surface coverage of $\mathrm{sp}^{2}$ carbon bonding decreased with an increase in the amount of nitrogen functional groups, thus leading to the red shift. Accordingly, the higher the amidation level, the suppressed the $\pi^{*}-\pi$ transition became. On the basis of the above deduction, this work offered one feasibility that the color-tunable emission from blue to greenish color and the increase of absorption in visible region were feasible if tuning to an appropriate amidation level on the GQD specimens.

\section{Acknowledgements}

The authors are very grateful for the support from the Ministry of Science and Technology (Taiwan, R.O.C.) under the contracts MOST 105-2628-E-155-002-MY3, MOST 105-2221-E-155-014MY3, MOST 105-2622-E-155-011-CC2, and MOST 105-2218-E155-007.

\section{References}

1 Y. N. Guo, X. Lu, J. Weng and Y. Leng, J. Phys. Chem. C, 2013, 117, 5708-5717.

2 S. Biswas and L. T. Drzal, Nano Lett., 2009, 9, 167-172. 
3 C. T. Hsieh, J. L. Gu, Y. C. Chen and D. Y. Tzou, Electrochim. Acta, 2013, 98, 39-47.

4 S. M. Paek and E. Yoo, Nano Lett., 2009, 9, 72-75.

5 C. T. Hsieh, W. Y. Lee, C. E. Lee and H. Teng, J. Phys. Chem. C, 2014, 118, 15146-15153.

6 P. Huang, J. J. Shi, M. Zhang, X. H. Zhong, Y. M. Ding, X. Cao, M. Wu and J. Lu, J. Phys. Chem. Lett., 2016, 7, 2888-2892.

7 M. H. Jang, H. D. Ha, E. S. Lee, F. Liu, Y. H. Kim, T. S. Seo and Y. H. Cho, Small, 2015, 11, 3773-3781.

8 L. H. Poh, P. Simek, Z. Sofer and M. Pumera, ACS Nano, 2013, 7, 5262-5272.

9 S. A. Hasan, E. K. Tsekoura, V. Sternhagen and M. Stromme, J. Phys. Chem. C, 2012, 116, 6530-6536.

10 B. Guo, Q. Liu, E. Chen, H. Zhu, L. Fang and J. R. Gong, Nano Lett., 2010, 10, 4975-4980.

11 H. Tetsuka, R. Asahi, A. Nagoya, K. Okamoto, I. Tajima, R. Ohta and A. Okamoto, Adv. Mater., 2012, 24, 5333-5338.

12 G. S. Kumar, R. Roy, D. Sen, U. K. Ghorai, R. Thapa, N. Mazumder, S. Saha and K. K. Chattopadhyay, Nanoscale, 2014, 6, 3384-3391.

13 J. Ortiz-Medina, F. Lopez-Urias, H. Terrones, F. J. RodriguezMacias, M. Endo and M. Terrones, J. Phys. Chem. C, 2015, 119, 13972-13978.

14 J. Choi, H. Lee, K. Kim, B. Kim and S. Kim, J. Phys. Chem. Lett., 2010, 1, 505-509.

15 N. Peimyoo, J. Li, J. Shang, X. Shen, C. Qiu, L. Xie, W. Huang and T. Yu, ACS Nano, 2012, 6, 8878-8886.

16 J. Liu, H. Yang, S. G. Zhen, C. K. Poh, A. Chaurasia, J. Luo, X. Wu, E. K. L. Yeow, N. G. Sahoo, J. Lin and Z. Shen, RSC Adv., 2013, 3, 11745-11750.

17 P. K. M. K. S. Shanthini and C. Srivastava, $R S C A d v .$, 2015, 5, 53865-53869.

18 X. Huang, S. Li, Z. Qi, Z. Wei, Y. Wei and Y. Fang, Nanotechnolgy, 2015, 26, 105602.

19 C. T. Hsieh and J. H. Huseh, RSC Adv., 2016, 6, 64826-64831.

20 S. H. Jin, D. H. Kim, G. H. jun, S. H. Hong and S. Jeon, ACS Nano, 2013, 7, 1239-1245.

21 M. Xie, Y. Su, X. Lu, Y. Zhang, Z. Yang and Y. Zhang, Mater. Lett., 2013, 93, 161-164.
22 Y. Dong, J. Shao, C. Chen, H. Li, R. Wang, Y. Chi, X. Lin and G. Chen, Carbon, 2012, 50, 4738-4743.

23 S. Kim, S. W. Hwang, M. K. Kim, D. Y. Shin, D. H. Shin, C. O. Kim, S. B. Yang, J. H. Park, E. Hwang, S. H. Choi, G. Ko, S. Sim, C. Sone, H. J. Choi, S. Bae and B. H. Hong, ACS Nano, 2012, 6, 8203-8208.

24 L. C. Chen, C. Y. Teng, C. Y. Lin, H. Y. Chang, S. J. Chen and H. Teng, Adv. Energy Mater., 2016, 6, 1600719.

25 J. Lu, J. X. Yang, J. Wang, A. Lim, S. Wang and K. P. Loh, ACS Nano, 2009, 3, 2367-2375.

26 S. Sarkar, M. Sudolska, M. Dubecky, C. J. Reckmeier, A. L. Rogach and R. Zboril, J. Phys. Chem. C, 2016, 120, 1303-1308.

27 S. Ravula, S. N. Baker, G. Kamath and G. A. Baker, Nanoscale, 2015, 7, 4338-4353.

28 K. Parvez, Z. S. Wu, R. Li, X. Liu, R. Graf, X. Feng and K. Müllen, J. Am. Chem. Soc., 2014, 136, 6083-6091.

29 T. F. Yeh, C. Y. Teng, S. J. Chen and H. Teng, Adv. Mater., 2014, 26, 3297-3303.

30 H. Wang, T. Maiyalagan and X. Wang, ACS Catal., 2012, 2, 781-794.

31 T. F. Yeh, S. J. Chen and H. Teng, Nano Energy, 2015, 12, 476485.

32 B. Guo, Q. Liu, E. Chen, H. Zhu, L. Fang and J. R. Gong, Nano Lett., 2010, 10, 4975-4980.

33 T. F. Yeh, W. L. Huang, C. J. Chung, I. T. Chiang, L. C. Chen, H. Y. Chang, W. C. Su, C. Cheng, S. J. Chen and H. Teng, J. Phys. Chem. Lett., 2016, 7, 2087-2092.

34 G. Eda, Y. Y. Lin, C. Mattevi, H. Yamaguchi, H. A. Chen, I. S. Chen, C. W. Chen and M. Chhowalla, Adv. Mater., 2010, 22, 505-509.

35 F. liu, M. H. Jang, H. D. Ha, J. H. Kim, Y. H. Cho and T. S. Seo, Adv. Mater., 2013, 25, 3657-3662.

36 S. Zhu, J. Zhang, S. Tang, S. Qiao, L. Wang, H. Wang, X. Liu, B. Li, Y. Li, W. Yu, X. Wang, H. Sun and B. Yang, Adv. Funct. Mater., 2012, 22, 4732-4740.

37 J. Shen, Y. Zhu, C. Chen, X. Yang and C. Li, Chem. Commun., 2011, 47, 2580-2582. 